\title{
Does antimicrobial use density at the ward level influence monthly central line-associated bloodstream infection rates?
}

This article was published in the following Dove Press journal:

Infection and Drug Resistance

I December 2014

Number of times this article has been viewed

\author{
Junichi Yoshida \\ Yukiko Harada \\ Tetsuya Kikuchi \\ Ikuyo Asano \\ Takako Ueno \\ Nobuo Matsubara \\ Infection Control Committee, \\ Shimonoseki City Hospital, \\ Shimonoseki, Japan
}

Correspondence: Junichi Yoshida Infection Control Committee, Shimonoseki City Hospital,

I-I3-I Koyo-cho, Shimonoseki 750-8520, Japan

$\mathrm{Tel}+8 \mid 8323$ I 4 I I I

Fax +8I 832243838

Email yoshidaj@uicalumni.org

\begin{abstract}
The aim of this study was to elucidate risk factors, including ward antimicrobial use density (AUD), for central line-associated bloodstream infection (CLABSI) as defined by the Centers for Disease Control and Prevention in a 430-bed community hospital using central venous lines with closed-hub systems. We calculated AUD as (total dose)/(defined daily dose $\times$ patient days) $\times 1,000$ for a total of 20 drugs, nine wards, and 24 months. Into each line day data, we inputed AUD and device utilization ratios, number of central line days, and CLABSI. The ratio of susceptible strains in isolates were subjected to correlation analysis with AUD. Of a total of 9,997 line days over 24 months, CLABSI was present in 33 cases (3.3\%), 14 (42.4\%) of which were on surgical wards out of nine wards. Of a total of 43 strains isolated, eight (18.6\%) were methicillin-resistant Staphylococcus aureus (MRSA); none of the MRSA-positive patients had received cefotiam before the onset of infection. Receiver-operating characteristic analysis showed that central line day 7 had the highest accuracy. Logistic regression analysis showed the central line day showed an odds ratio of 5.511 with a 95\% confidence interval of 1.936-15.690 as did AUD of cefotiam showing an odds ratio of 0.220 with $95 \%$ confidence interval of $0.00527-0.922$ $(P=0.038)$. Susceptible strains ratio and AUD showed a negative correlation $\left(R^{2}=0.1897\right)$. Thus, CLABSI could be prevented by making the number of central line days as short as possible. The preventative role of AUD remains to be investigated.
\end{abstract}

Keywords: bloodstream infection, central line, antimicrobial use density

\section{Introduction}

Increasing dwell time of a central venous line is known to increase the risk of central line-associated bloodstream infection (CLABSI). Of late, substandard care of central lines because of the burden of workload has been identified as another risk factor. ${ }^{1}$ Prolonged use of broad-spectrum antibiotics ${ }^{2}$ has also been reported to predispose to CLABSI. However, the possible influence of antimicrobial use density (AUD) on CLABSI and the microbial susceptibility of isolates have seldom been investigated. In Japanese hospitals, a variety of classical antimicrobials are used, but their effect on CLABSI at the ward level has not been addressed, and the possible influence of AUD on CLABSI is unknown. AUD by ward and month may predict bloodstream infection in terms of space in the hospital and calendar time frames, respectively. In this study, we attempted to identify possible ways of preventing CLABSI, including AUD, by ward and month.

\section{Materials and methods}

\section{Central lines and sites of insertion}

This retrospective study was performed in our 430-bed community hospital from March 2011 through February 2013. Midline catheters, including central venous and 
Swan-Ganz catheters, were inserted under maximum sterile barrier conditions. Povidone iodine solution (10\%, Meiji Seika Pharma, Tokyo, Japan) was used as a skin antiseptic rather than $2 \%$ chlorhexidine, which was not commercially available in Japan. The infusion lines consisted of closed-hub systems that were disinfected using cotton swabs with 76.9-81.4 vol\% ethanol (Yoshida Pharmaceuticals, Tokyo, Japan), as described previously. ${ }^{3}$ The central lines used were the CV Legaforce SX (Terumo Co, Tokyo, Japan) and the Arrow central venous catheter (Telefex Inc, Limerick, PA, USA), with a SurePlug infusion set (Terumo Co) for the hubs.

The site of central line insertion was covered with a sterile transparent dressing that was replaced every 7 days. When a patient developed a fever of more than $38.0^{\circ} \mathrm{C}$, blood cultures were submitted; when possible, midline catheters were withdrawn and their tips were sent for culture. Although recommended by the Infectious Diseases Society of America, ${ }^{2}$ no blood cultures were drawn via the central line hubs. If the catheter was exchanged, continuous central line days were counted from 1 reduced from continuously counted line days.

Throughout the study period, infection control specialists inspected the wards weekly for compliance with techniques for prevention of contact infection and use of alcohol for hand hygiene. The central line infusates were prepared in the hospital pharmacy except in emergencies. Nurses on all wards were trained to inspect the sites of central line insertion in addition to monitoring body temperature in all patients. Thus, infection control measures were kept constant across the wards.

\section{Regulation of antimicrobial use}

The infection control committee regulated agents targeting methicillin-resistant Staphylococcus aureus (MRSA) and broadspectrum agents such as carbapenems, cefozopran, and cefepime. Otherwise, antimicrobials were administered at the discretion of the attending physicians. However, prophylactic use of agents for MRSA was only allowed on confirmation of MRSA by active surveillance culture prior to cardiovascular and orthopedic surgical procedures involving placement of an implant.

\section{Definition of central line-associated bloodstream infection}

We defined CLABSI in accordance with the definition of the Centers for Disease Control and Prevention. ${ }^{4}$ Thus, cases with infectious foci other than in the bloodstream were not identified as CLABSI and did not require central line withdrawal or replacement. Likewise, positive cultures from blood or other specimens in the presence of infectious foci other than in the bloodstream were not deemed to be CLABSI.

\section{Definition of antimicrobial use density}

The AUD was defined for 20 antimicrobials by ward and month as follows:

$$
\mathrm{AUD}=(\text { Total dose }) /(\mathrm{DDD} \times \text { Patient-days }) \times 1,000
$$

where DDD is the daily dose as defined by the World Health Organization. ${ }^{5}$ The AUD data were averaged for the preceding month and for number of central line days in the analysis.

\section{Susceptibility and its correlation with AUD}

Susceptibility of microbes isolated in CLABSI was defined according to the guideline of the Clinical and Laboratory Standards Institute (Wayne, PA, USA). For a given antimicrobial agent, the ratio of susceptible strains to all the strains was calculated and subjected to correlation analysis with the mean values of the corresponding AUD. Should only one strain have susceptibility for any drug, the ratio of susceptible strains was excluded from the analysis.

\section{Definition of device utilization ratio}

To evaluate the workload associated with use of a central line, the device utilization (DU) ratio $^{6}$ was determined as follows for each ward:

DU ratio $=$ Number of central line days/Patient days

where the time interval was from either March 2011 to February 2012 or March 2012 to February 2013.

\section{Statistical analysis}

Into each item of data on central line day, we entered patient sex and age, the mean AUD for the month and the preceding month, the DU ratio, continuous days of central line placement, and presence or absence of CLABSI. To determine the optimal threshold for logistic regression analysis, a receiver-operating characteristic analysis was performed for continuous data, including central line days. We then performed a univariate logistic regression analysis of background factors for CLABSI, which was the outcome factor in this analysis. Statistical significance was determined as $P<0.05$. Factors significant in the univariate analysis were subsequently tested by multivariate regression analysis. When $P$-values could not be determined because of sample deviation, those factors were excluded for further analysis. Statistical Package for the Social Sciences software (IBM Corporation, Armonk, NY, USA) was used for the statistical analysis. 


\section{Ethical considerations}

This study was approved by the internal review board at our institution. This decision was based on its compliance with the Ethical Guideline on Epidemiological Research (Revised April 1, 2013) issued by the Ministry of Education, Culture, Sports, Science, and Technology and by the Ministry of Health and Welfare, Japan.

\section{Results}

\section{Overview and clinical background}

Of a total of 9,997 line days over 24 months, CLABSI was present in 33 cases (3.3\%), 14 (42.4\%) of which were in surgery ward out of a total of nine wards. Among the positive patients, eight (24.2\%) had malignancy, eight (24.2\%) had pneumonia, three (9.1\%) had urinary tract infection, three $(9.1 \%)$ had secondary peritonitis, two (6.1\%) had joint infection, two (6.1\%) had pancreatitis, two (6.1\%) had vascular disease, and other diseases were present in five (15.2\%) cases. These patients had received a variety of antimicrobials prior to the onset of CLABSI but none were given cefotiam (Table 1). Prior to the episode of CLABSI with isolation of MRSA as described in the following, two patients had received vancomycin and one had received linezolid.

\section{Bacterial isolates}

Of 43 strains isolated as CLABSI, S. aureus accounted for ten cases (including eight MRSA strains), Candida parapsilosis for seven, Enterobacter cloacae for four, Bacillus cereus for three, Enterococcus faecalis for three, Staphylococcus epidermidis for three, Candida glabrata for two, Staphylococcus caprae for two, Staphylococcus warneri for two, and another seven species in one case each. No strains carried extended spectrum beta-lactamase.

\section{Receiver-operating characteristic analysis}

The receiver-operating characteristic analysis revealed central line day 7 had the highest accuracy. Univariate regression analysis showed significance in central line day and AUD of cefotiam. However, the ward factor, DU ratio, and others did not show statistical significance. Multivariate regression analysis showed central line day and AUD of cefotiam had an odds ratio of $5.511(P<0.001)$ and $0.220(P=0.038)$, respectively.

\section{Susceptible strain ratios and AUD}

A total of ten pairs of susceptible strain ratios and AUDs were subjected to correlation analysis, including ampicillin, cefazolin, cefmetazole, cefotaxime, cefotiam, clindamycin, flomoxef, fosfomycin, gentamicin, and imipenem/cilastatin. The pairs were inversely correlated, with $R^{2}=0.1897$ (Figure 1).
Table I Antimicrobials given prior to the onset of central lineassociated bloodstream infection ( $n=33$ cases)

\begin{tabular}{|c|c|c|}
\hline & Total dose $(\mathrm{g})$ & Cases \\
\hline & Median (range) & \\
\hline Ampicillin-sulbactam & $9(7.5-10)$ & $n=4$ \\
\hline Cefazolin & $6(2-6)$ & $n=3$ \\
\hline Cefmetazole & $24(24)$ & $n=1$ \\
\hline Cefoperazole-sulbactam & $9(8-10)$ & $n=2$ \\
\hline Ceftazidime & $28(2-38)$ & $n=3$ \\
\hline Ceftriaxone & $6(2-14)$ & $n=3$ \\
\hline Ciprofloxacin & $0.6(0.6)$ & $n=1$ \\
\hline Clindamycin & $8.4(8.4-8.4)$ & $n=2$ \\
\hline Fluconazole & $\mathrm{I} .0(\mathrm{I} .0)$ & $n=1$ \\
\hline Flomoxef & I8 (8-28) & $n=2$ \\
\hline Imipenem-cilastatin & $3.5(3.5)$ & $n=1$ \\
\hline Levofloxacin & $6.0(6.0)$ & $n=1$ \\
\hline Linezolid & $17.6(8.4-16.8)$ & $\mathrm{n}=2$ \\
\hline Meropenem & $5.5(4-14)$ & $\mathrm{n}=5$ \\
\hline Minocycline & $8.4(8.4)$ & $\mathrm{n}=\mathrm{I}$ \\
\hline Piperacillin & II (4-14) & $\mathrm{n}=2$ \\
\hline Piperacillin-tazobactam & 189 (189-189) & $\mathrm{n}=2$ \\
\hline Sulfamethoxazole/trimethoprim & $16 / 3.2(16) /(3.2)$ & $n=1 / 1$ \\
\hline Teichoplanin & $12(12)$ & $n=1$ \\
\hline Vancomycin (parenteral) & I $(0.5-10)$ & $n=3$ \\
\hline Subtotal & & $\mathrm{n}=42^{\mathrm{a}}$ \\
\hline None & & $\mathrm{n}=5^{\mathrm{b}}$ \\
\hline Total & & $\mathrm{n}=47$ \\
\hline
\end{tabular}

In seven strains tested for susceptibility to cefotiam, two $(28.6 \%)$ were susceptible.

\section{Discussion}

The current study shows that an increased AUD for cefotiam may help prevent CLABSI. The possible effect of ward-level antimicrobial pressure on CLABSI has not been addressed previously. To investigate this potential wardspecific effect, we used ward AUDs of various antimicrobials, in which cefotiam had significant odds ratio to decrease CLABSI. Nakamura et $\mathrm{al}^{7}$ reported that methicillin-resistant S. epidermidis isolated from blood culture and central lines had a low minimum inhibitory concentration for cefotiam. The definition of CLABSI would hardly permit $S$. epidermidis as a causative microbe but $S$. epidermidis remains as one of the major causes. Anecdotally cefotiam, not marketed in the USA, ${ }^{8}$ has seldom been described in the literature on bloodstream infection.

At the level of the individual patient, cefotiam was susceptible in a limited number of cases, thus precluding its empirical use ${ }^{9}$ in suspected cases of CLABSI. 
Table 2 Logistic regression analyses on the risk factors for CLABSI, including ward-specific AUD of multiple agents

\begin{tabular}{|c|c|c|c|c|}
\hline Factor & Univariate analysis & $P$-value & Multivariate analysis & $P$-value \\
\hline \multicolumn{5}{|l|}{ AUDs } \\
\hline Ampicillin & $0.26(0.06-1.07)$ & 0.06 & & \\
\hline Ampicillin-sulbactam & $0.70(0.35-1.39)$ & 0.31 & & \\
\hline Cefazolin & $1.76(0.88-3.55)$ & 0.11 & & \\
\hline Cefmetazole & $0.69(0.24-1.95)$ & 0.47 & & \\
\hline Ceftazidime & $0.68(0.33-I .4 I)$ & 0.31 & & \\
\hline Cefoperazole-sulbactam & $1.46(0.5 I-4.16)$ & 0.48 & & \\
\hline Cefotaxim & $1.14 \times 10^{-8}\left(1.14 \times 10^{-8}-1.14 \times 10^{-8}\right)$ & N/A & & \\
\hline Cefotiam & $0.21(0.05-0.89)$ & $0.03 *$ & $0.22(0.05-0.92)$ & $0.04 *$ \\
\hline Cefmetazole & $0.69(0.24-1.95)$ & 0.48 & & \\
\hline Cefozopran & $0.73(0.3 \mathrm{I}-\mathrm{I} .79)$ & 0.50 & & \\
\hline Cefpirome & $1.06 \times 10^{-7}\left(1.06 \times 10^{-7}-1.06 \times 10^{-7}\right)$ & $\mathrm{N} / \mathrm{A}$ & & \\
\hline Ceftriaxone & $0.41(0.16-1.08)$ & 0.07 & & \\
\hline Clindamycin & $0.62(0.31-1.24)$ & 0.18 & & \\
\hline Flomoxef & $0.87(0.43-1.74)$ & 0.68 & & \\
\hline Fosfomycin & I.0I (0.48-2.08) & 0.99 & & \\
\hline Gentamicin & $1.34(0.67-2.68)$ & 0.40 & & \\
\hline Imipenem-cilastatin & $1.86(0.83-4.13)$ & 0.13 & & \\
\hline Linezolid & $1.89(0.95-3.76)$ & 0.07 & & \\
\hline Meropenem & $0.65(0.30-1.39)$ & 0.26 & & \\
\hline Minocycline & $1.34(0.68-2.67)$ & 0.40 & & \\
\hline Panipenem-betamipron & $0.78(0.23-2.56)$ & 0.68 & & \\
\hline Piperacillin & $0.87(0.43-1.73)$ & 0.69 & & \\
\hline Piperacillin-tazobactam & I.87 (0.9I-3.86) & 0.09 & & \\
\hline Vancomycin & $1.16(0.58-2.31)$ & 0.66 & & \\
\hline Surgical ward & $1.40(0.70-2.80)$ & 0.34 & & \\
\hline CL-day & $5.60(1.97-15.94)$ & $<0.01 *$ & $5.51(1.94-15.69)$ & $<0.01 *$ \\
\hline Male sex & $0.62(0.29-1.35)$ & 0.23 & & \\
\hline Age $>75$ years & $0.90(0.45-1.8 I)$ & 0.76 & & \\
\hline DU ratio & I.43 (0.72-2.86) & 0.30 & & \\
\hline
\end{tabular}

Notes: $* P<0.05$. DU ratio is defined as number of CL-days/number of patient-days.

Abbreviations: AUD, antimicrobial use density; CLABSI, central line-associated bloodstream infection; N/A, not available due to sample deviation; CL-day, days of central line placement; DU, device utilization of the former half as the index study period.

As regards MRSA, three of eight cases had received antimicrobial agents for MRSA but developed CLABSI, due probably to biofilm coated on the central line. At the ward level of antimicrobial use, however, less commonly

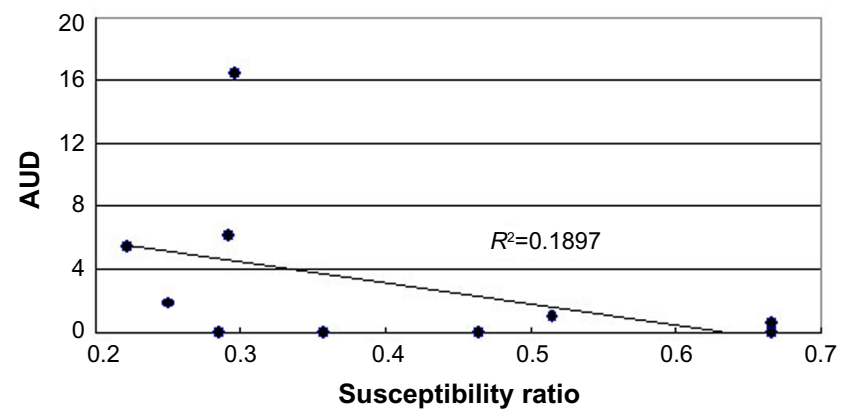

Figure I Correlation between susceptibility ratio (horizontal axis) defined as the number of susceptible strains divided by the number of all strains undergoing susceptibility versus the median values for antimicrobial use density (AUD, vertical axis). They show a negative correlation (superimposed line) with $R^{2}=0.1897$ for ten drugs available indicating increase in AUD was associated with decrease in susceptibility of the same antimicrobial. prescribed antimicrobials may have a preventive effect on CLABSI.

A literature search for a possible relationship between AUD and CLABSI failed to yield similar studies. However, Meyer et a ${ }^{10}$ reported that increased AUDs for glycopeptides and quinolones showed increased hazard ratios with CL rates per 1,00 patient days in intensive care units in Germany. Kanerva et $\mathrm{a} \mathrm{l}^{11}$ proposed a patient case-mix adjustment model for antibiotic use in Finnish acute care hospitals, suggesting that use of central lines was not a significant risk factor in the model. Thus, correlation between ward-specific and monthspecific AUD and CLABSI has rarely been addressed.

In Japan, Ikeda et $\mathrm{a}^{12}$ reported that a permission system for use of carbapenems reduced their AUD and the incidence of drug-resistant bacteria. Similarly, our study showed that AUDs were inversely correlated with susceptible strain ratios, indicating that increased use of any antimicrobial may decrease susceptibility for that particular drug. It has been 
reported that a prolonged duration of central line placement is a risk factor for CLABSI, ${ }^{13}$ and this was confirmed in the present study. Trick et $\mathrm{al}^{14}$ recommended that the need for a central line should be assessed on a day-by-day basis and that nursing staff should be encouraged to notify physicians of unnecessary central lines. The DU ratio was investigated as a possible risk factor for CLABSI, but no association was found. This may indicate that care at handling central lines was constantly observed regardless of the workload as represented by DU ratio. It has been suggested that male patient sex is a significant risk factor for CLABSI, ${ }^{1}$ but this was not the case in our study, and nor was being elderly.

The limitations of the present study are its retrospective design and the fact that the data are derived from a single institution. Another limitation is the lack of validation of computation of the background factor of AUDs averaging the reference and previous months for a central line day. Our findings require validation by further studies in the future.

\section{Disclosure}

JY has received personal fees from Merck and Co Inc (Whitehouse Station, NJ, USA), Astellas Pharma Inc (Tokyo, Japan), Bayer Holding (Tokyo, Japan), and Dainippon Sumitomo Pharma Inc (Tokyo, Japan). The other authors report no conflicts of interest in this work.

\section{References}

1. Marschall J, Mermel LA, Classen D, et al. Strategies to prevent central line-associated bloodstream infections in acute care hospitals. Infect Control Hosp Epidemiol. 2008;29 Suppl 1:S22-S30.

2. Mermel LA, Allon M, Bouza E, et al. Clinical practice guidelines for the diagnosis and management of intravascular catheter-related infection: 2009 Update by the Infectious Diseases Society of America. Clin Infect Dis. 2009;49:1-45.

3. Yoshida J, Ishimaru T, Fujimoto M, Hirata N, Matsubara N, Koyanagi N. Risk factors for central venous catheter-related bloodstream infection: a 1073-patient study. J Infect Chemother. 2008;14:399-403.
4. Horan TC, Andrus M, Dudeck MA. CDC/NHSN surveillance definition of health care-associated infection and criteria for specific types of infections in the acute care setting. Am J Infect Control. 2008;36:309-332.

5. WHO Collaborating Centre for Drug Statistics Methodology. Norwegian Institute of Public Health ATC/DDD Index 2013. Geneva, Switzerland: World Health Organization. Available from: http://www.whocc.no/ atc_ddd_index/.Accessed September 3, 2014.

6. Murphy C, Andrus M, Barnes S, et al. APIC elimination guide: guide to the elimination of catheter-related bloodstream infections. Washington, DC, USA: Associations for Professionals in Infection Control and Epidemiology. Available from: http://www.apic.org/ Resource_/EliminationGuideForm/259c0594-17b0-459d-b395fb $143321414 \mathrm{a} /$ File/APIC-CRBSI-Elimination-Guide.pdf. Accessed September 3, 2014.

7. Nakamura A, Oguri T, Misawa S, et al. In vitro activity of cefotiam against oxacillin-resistant Staphylococcus epidermidis strains reevaluation of beta-lactam antibiotics efficiency on MRSE. Jpn $J$ Antibiot. 2007;60:153-160.

8. Bratzler DW, Dellinger EP, Olsen KM, et al. Clinical practice guidelines for antimicrobial prophylaxis in surgery. Am J Health Syst Pharm. 2013;70:195-283.

9. Munson EL, Diekema DJ, Beekmann SE, Chapin KC, Doern GV. Detection and treatment of bloodstream infection: laboratory reporting and antimicrobial management. J Clin Microbiol. 2003;41:495-497.

10. Meyer E, Schwab F, Jonas D, Rueden H, Gastmeier P, Daschner FD. Surveillance of antimicrobial use and antimicrobial resistance in intensive care units (SARI): 1. Antimicrobial use in German intensive care units. Intensive Care Med. 2004;30:1089-1096.

11. Kanerva M, Ollgren J, Lyytikäinen O; Finnish Prevalence Survey Study Group. Benchmarking antibiotic use in Finnish acute care hospitals using patient case-mix adjustment. $J$ Antimicrob Chemother. 2011;66:2651-2654

12. Ikeda $Y$, Mamiya T, Nishiyama $H$, et al. A permission system for carbapenem use reduced incidence of drug-resistant bacteria and cost of antimicrobials at a general hospital in Japan. Nagoya J Med Sci. 2012;74:93-104.

13. Yoshida J, Ishimaru T, Kikuchi T, et al. Central line-associated bloodstream infection: is the hospital epidemiology of methicillin-resistant Staphylococcus aureus relevant? J Infect Chemother. 2010;16:33-37.

14. Trick WE, Vernon MO, Welbel SF, Wisniewski MF, Jernigan JA, Weinstein RA. Unnecessary use of central venous catheters: the need to look outside the intensive care unit. Infect Control Hosp Epidemiol. 2004;25:266-268.
Infection and Drug Resistance

\section{Publish your work in this journal}

Infection and Drug Resistance is an international, peer-reviewed openaccess journal that focuses on the optimal treatment of infection (bacterial, fungal and viral) and the development and institution of preventive strategies to minimize the development and spread of resistance. The journal is specifically concerned with the epidemiology of antibiotic

\section{Dovepress}

resistance and the mechanisms of resistance development and diffusion in both hospitals and the community. The manuscript management system is completely online and includes a very quick and fair peerreview system, which is all easy to use. Visit http://www.dovepress.com/ testimonials.php to read real quotes from published authors. 